\title{
Christliche Kommunikation als prophetische Aufgabe
}

\author{
von Hans Florin
}

Prognose oder Prophetie, - das ist hier die Frage! Eine Prognose ist einfach zu geben: Auch im Jahr 2000 wird die Kirche Jesu Christi mit der Welt und in der Welt kommunizieren. Eine prophetische Aussage ist schon schwieriger. Wird die Kirche auf Gottes Geheiß mit der Welt kommunizieren, diese Welt auf Gottes Willen aufmerksam machen, ihr Gottes Gebot und Auftrag ausrichten, oder wird sie mit sich selber reden? Mit der Welt im Auftrag Gottes reden ist prophetische Kommunikation. In der Welt mit sich selbst reden ist vieles, was seit eh und je und auch sicherlich noch im Jahre 2000 christliche Kommunikation ausmacht.

Eine Reflektion über "Christliche Kommunikation im Jahr 2000" wird daher sowohl prognostizierende wie auch prophetische Elemente enthalten. Eine solche Reflektion aus Anlaß des zehnjährigen Bestehens der Zeitschrift "Communicatio Socialis" sollte aber das prophetische Element nicht auslassen.

Prophetische Kommunikation wird nicht von der Kirche bestimmt. Sie wird von Gott gefordert, befohlen:

"Aber du, Menschenkind, höre du, was ich dir sage, und sei nicht ungehorsam, wie das ungehorsame Haus ist. Tue deinen Mund auf und iß, was ich dir geben werde. Und ich sah, und siehe, da war eine Hand gegen mich ausgestreckt, die hatte einen zusammengelegten Brief; den breitete sie aus vor mir, und er war beschrieben auswendig und inwendig, und stand darin geschrieben Klage, Ach und Wehe. Und er sprach zu mir: Du Menschenkind, $i ß$, was vor dir ist, iß diesen Brief, und gehe hin und predige dem Hause Israel" (Hesekiel 2,8-3,1).

Der Prophet sucht sich sein Amt nicht. Er möchte ihm vielmehr ausweichen. Der Brief, die Botschaft, die er zu kommunizieren hat, ist keine bequeme Nachricht, keine bestätigende Information. Christliche Kommunikation hat immer in dieser Spannung zwischen Widerstand gegen die Welt und Ergebung an die Welt gestanden. Sie wird auch weiter in dieser Spannung bleiben. Sie muß sogar in dieser Spannung bleiben muß sich in ihr erfüllen. Ihre Aufgabe ist, in der Kirche und über die Kirche zu informieren. Ihr Auftrag ist aber auch, in die Welt hineinzusagen, welche Absichten Gott mit seiner Welt hat, zu welchem $Z_{w}$ edk er sie geschaffen hat, mit welcher Geduld, aber auch mit welchem Gericht er sie verfolgt, und warum er seinen Sohn Jesus Christus für die Welt und ihre Menschen geopfert und Ihn verherrlicht hat.

Christliche Kommunikation hat sich dieser Aufgabe und diesem Auftrag gestellt. Sie ist $\mathrm{ihm}$ aber auch immer wieder ausgewichen. Im Durchstehen der Spannung zwischen Widerstand und Ergebung ist christliche Kommunikation nicht besser, erfolgreicher gewesen als die Kirche selbst.

Dr. Hans W. Florin ist Exekutiv-Direktor der Weltvereinigung für Christliche Kommunikation (WACC), London. 
Predigt, Seelsorge, Armenhilfe, Krankenpflege, theologische Lehre und ihre Anwendung, ekklesiale Organisation und politische Orientierung finden immer in dieser Spannung statt. Und die, die dank ihrer besonderen Gaben über dieses kirchliche Tun berichten, haben in ihrer Existenz als Kommunikatoren Anteil an dieser Spannung. Sie werden sogar zunehmend durch diese Spannung belastet werden, denn die Kirche verliert mehr und mehr Kontrolle über die Medien der Kommunikation, die sie im Frühstadium der Medienentwicklung einmal ausgeübt hatte. Moderne Staaten bedürfen nicht mehr der Kirche und ihrer Organe, um ihre Verwaltungsstrukturen zu vervollkommnen. Modernes Wissen wird allenfalls in Klöstern begleitet, selten noch gefördert. Bildung und Erziehung sind in die Verantwortung der nach politischen oder wirtschaftlichen Gesichtspunkten gesteuerten Staaten übergegangen. Auch die neuen Nationalstaaten der Dritten Welt übernehmen diese Verantwortung in ihre eigene Regie. Krankenpflege, Sozialfürsorge und Gesundheitswesen sind weitgehend säkularisiert worden. Politische Stellungnahmen der Kirche sind zumeist nicht sehr erwünscht und werden oft und gern von Kirchenleitungen gemieden, um das säkular geordnete Gemeinwohl nicht zu stören. Das christliche Buch muß sich verkaufen lassen. Die kirchliche Nachricht muß einen vermarktbaren Aspekt haben. Der christliche Film-, Funk- oder Fernsehproduzent hat weithin nur noch sein Gewissen, das ihn berät in seiner Spannung zwischen kirchlichen Interessen und dem Anspruch, den seine ihn beschäftigende öffentlich-rechtliche Kommunikationsanstalt an ihn stellt. Es ist beobachtet worden, daß dort, wo die Kirche die Kommunikationsmedien nicht oder nicht mehr kontrolliert, sie ein gebrochenes Verhältnis zur Kommunikation entwickelt hat.

Aus der Erfahrung im Bereich der World Association for Christian Communication (WACC) lassen sich etwa folgende Trends in der christlichen Medienarbeit ablesen: Kommunikation wird für die Kirche immer bedeutender, will sie neben der anschwellenden Kommunikationsflut aus Politik und Wirtschaft ihre Botschaft vorzeigen und ihre Glaubwürdigkeit bekräftigen. Die Art ihrer Kommunikation wird nach Programmformat und Mediengebrauch schon heute weitgehend von den Gesetzen bestimmt, die die Massenmedien steuern. Diese Gesetze sind von den Bedürfnissen der Wirtschaft und Politik geprägt - weniger von der Kirche. Die Kirche hatte nach dem Ausbruch der Neuzeit kaum noch Einfluß auf die Medienentwicklung - weniger noch auf die Entwicklung der Massenmedien. Dennoch hat es die Kirche sich nicht leisten können, ihrerseits auf den Gebrauch der Massenmedien zu verzichten. Die Frage nach der Absatzmöglichkeit kirchlicher Inhalte wird bestimmt von den Interessen der Medieneigner. Und wo die Kirche selbst Medieneigner ist, wie bei Verlagshäusern, Periodika, Tageszeitungen, Rundfunkstudios und -stationen oder Filmproduktionsstätten, muß sie sich auf die Gesetze des Medienmanagement einstellen, wenn sie erreichen will, daß ihre angebotenen Inhalte in Konkurrenz mit anderen Inhalten von dem zu beschränkter Aufnahme befähigten Medienverbraucher aufgegriffen werden. Sorgfältiges Medienmanagement ist daher auch für kirchliche Kommunikation in Zukunft von größter Bedeutung.

Angesichts der Tatsache, daß die säkular geprägten Mediengesetzlichkeiten schließlich auch die Kommunikationsinhalte beeinflussen, muß die Kirche sich bis zum Jahr 2000 zunehmend auch der Frage stellen, ob sie und gegebenenfalls wie sie sich der Massenmedien bedienen kann und darf. Vor dieser Frage stehen die reichen Kirchen, die sich ihre eigenen massenmedialen Produktionsstätten leisten können, ebenso wie 
die armen Kirchen, vornehmlich in der Dritten Welt, die auf Zusammenarbeit mit von ihnen nicht kontrollierten Produktionsmitteln angewiesen sind.

Der Hinweis, daß die Existenz der lebendigen Glaubensgemeinde den kirchlichen Gebrauch der Massenmedien rechtfertige, wird im Jahr 2000 wahrscheinlich noch weniger überzeugend sein, als er es heute bereits ist. Denn schon heute ist die Glaubensgemeinde nicht mehr die Ortsgemeinschaft. In der Parochie wurde geistliches und weltliches Leben ungebrochen miteinander verarbeitet. Geburt, Leben, Tod, Leiden, Freude, Hoffnung - diesseitig und jenseitig - Verzweiflung, Krankheit, Fürsorge, Wohlergehen, Fleiß, Wohlstand, Mißgeschick und Armut erfuhren in der Parochie gleichermaßen Beachtung. Nicht mehr in der zur Norm werdenden städtischen Glaubensgemeinde. Diese Glaubensgemeinde wird bis zur Jahrhundertwende ihre von der Parochie unterschiedliche Dynamik weiter entwickeln: Gottesdienst und Eucharistie werden ihr Mittelpunkt sein. In der Ubernahme ausgewählter Sozialverpflichtungen in Mission und Diakonie wird sich ihr Selbstverständnis ausprägen. In Spiel, Unterricht und Gruppenarbeit wird der Heimatboden für die einzelnen Glieder der Glaubensgemeinde zubereitet. Kirchliche Kommunikation kommt in diese Glaubensgemeinde nur noch als Fach- und Spezialinformation hinein. Für das globale Zeugnis der Kirche durch die Massenmedien wird diese Glaubensgemeinde kaum mehr das geeignete Instrument zur Nacharbeit, zum Nachvollzug sein, so daß kirchliche Massenkommunikation, wie jede andere auch, eine Vielzahl von Individuen trifft, Christen wie Nicht-Christen, die das eine gemeinsam haben, daß sie sich nur mit Mühe konstruktiv mit den Inhalten kirchlicher Massenkommunikation auseinandersetzen können. Sie können lediglich passiv den Konsum verweigern, indem sie das christliche Buch nicht lesen, gar erst nicht kaufen, die religiöse Rundfunksendung abstellen, die kirchliche Wochenpresse unbeachtet lassen. Für diese Art von Konsum hat die Kirche sich jedoch nicht um den Gebrauch der Massenmedien bemüht. Kirche will niemals nur unterhalten - ein proprium, für das sich die Massenmedien besonders gut eignen. Kirche will vornehmlich den Menschen, die Menschengemeinschaft verändern, umkehren, hinwenden zu Christus, in welchem Gott uns Seine Vorstellung menschlichen Lebens und menschlicher Erfüllung offenbart hat. Ob Massenmedien Menschen verändern können, wird nicht zuletzt auch von den Medientheoretikern selbst bezweifelt. Was aber hat dann die Kirche mit den Massenmedien zu schaffen? Diese Frage bleibt solange bestehen, bis Kirche in und neben der Glaubensgemeinde die kommunizierende Gemeinde entdeckt und entwickelt hat: Die dialog-fähige Gruppe.

Christliche Kommunikation setzt schon heute diese kommunizierenden, die die christliche Kommunikation herausfordernden Gruppen voraus. Ohne sie wird christliche Kommunikation in den Jahren rapider Kostensteigerung bis zur Jahrhundertwende zu einem Luxus, den sich dann selbst die reichsten Kirchen kaum noch werden leisten können. Mit diesen Gruppen wird christliche Kommunikation, die die Veränderung, die Bekehrung der Menschen zum Ziel hat, erst ihre wahre Erfüllung finden, denn es werden die miteinander kommunizierenden Christengemeinden sein, die die auf Zerstörung gerichteten Werte unserer gegenwärtigen Gesellschaft verändern, umorientieren können. Christliche Kommunikation ist also nicht vornehmlich ein Massenkommunikationsprozeß, sondern ein Vorgang, der dem Einzelnen die Möglichkeit gibt, sich mit möglichst vielen anderen auszutauschen, ihnen zu begegnen, von ihnen zu lernen, zusammen mit ihnen aus der eigenen Provinzialität zu 
entfliehen und mit ihnen zu verantwortlichen - weil christlichen - Entscheidungen zu kommen.

Christliche Gruppenkommunikation muß behutsam entdeckt und eingeibt werden. Sie ist kein christliches Derivat gruppendynamischer Prozesse, die sich zumeist auf bestehende Gruppen konzentrieren und die in diesen Gruppen vorhandenen Vorurteile verfestigen. Christliche Gruppenkommunikation ist vielmehr das Erleben christlicher Existenz mit Menschen der verschiedensten Glaubenstraditionen in unterschiedlichen kulturellen, rassischen und politischen Bindungen. Christliche Gruppenkommunikation kann der die Glaubenserkenntnis bekräftigende rhythmische Refrain in Afrika sein, der aussagekräftige stilistische Tanz Indiens oder Indonesiens, das Puppenspiel, die Gestik romanischer Menschen, das beruhigende MiteinanderSchweigen-Können der Finnen, das explusive Drama der Amerikaner in Nord und Sïd.

Dies Entfalten und Einüben selbst ist bereits christliche Kommunikation und wird im Jahr 2000 hoffentlich auch in den mit einer längeren Tradition befrachteten Kirchen des Westens beheimatet sein. In dieser Form christlicher Gruppenkommunikation bietet sich zugleich auch die Alternative an, die die kritische Anfrage an die bestehende kirchliche Massenkommunikationspraxis rechtfertigt. Ohne zu wissen, ob dristliche Gruppenkommunikation Menschen zu verändern vermag, sollte das Engagement der Kirche in der Massenkommunikation nicht abgebaut werden. Sollte es sich aber erweisen, daß die Gruppe das Informationsangebot aus der kirchlichen Massenkommunikation aufnehmen und zur Veränderung von Menschen umsetzen kann, wachsen der christlichen Kommunikationstechnik neue Aufgaben zu - Aufgaben, die etwa um die Jahrhundertwende zu meistern wären.

Die Kommunikationstechnik hat wie kaum eine andere zur Transparenz unserer Welt beigetragen: Telekommunikation überbrückt Kontinente. Der Rundfunk ist aus diesem interkontinentalen Kommunikationsbereich nicht mehr wegzudenken. Echte transkulturelle Kommunikation ist aber dem Rundfunk als einem EinbahnKonsuminstrument noch kaum gelungen. Telefon und Telex müssen für einen breiteren als den Individualverkauf erst weiterentwickelt werden. Doch die technischen Voraussetzungen für eine zwei- oder mehrbahnige Tele-Audiobildkommunikation über Satellitenverbindung sind schon gegeben. Die Wirtschaftlichkeit dieser Technik wird entwickelt werden. Die Industrie bereitet sich auf die Nutzung dieser vielseitigen Kommunikationsebene vor. Die Kirche muß in den kommenden Jahren diese Möglichkeit prüfen und zu einem Zeitpunkt, zu dem es nicht zu spät ist, entscheiden, ob sie teilhaben will und $\mathrm{zu}$ welchem speziellen Gebrauch. Sicherlich werden kirchliche Behörden ihre Konferenzbedürfnisse mit dem Angebot dieser Tele-Audiobildkommunikation befriedigen wollen, wenn erst einmal der Treibstoffmangel den heute so wirksam genutzten Flugverkehr unerschwinglich macht. Interessanter wäre aber noch der Gebrauch eines viel-kanaligen Instruments für die Förderung der christlichen Gruppenkommunikation, für eine Beheimatung der glaubenden Gemeinde in der urbanen Umwelt des 21. Jahrhunderts. Die Wünsche, die sich etwa für den Glaubens- und Erfahrungsaustausch der Kirchen und Gemeinden mit dem Afrikasender "Stimme des Evangeliums" vor dessen Nationalisierung in Athiopien verbunden haben, sowie das gediegene kirchliche Engagement in christlicher Massenkommunikation, das dort gepflegt worden ist, würden durch diese fortgeschrittene Kommunikationsform ihre Erfüllung erfahren. Nutzung einer solchen Tele-Audiobildkommunikation würde der Kirche zugleich den Zugang zu der für sie 
unaufgebbaren Gemeinde und zu kleinen Gruppen sichern und ihren Traum von einer auf Gespräch und Austausch ausgerichteten interkulturellen Massenkommunikation erfüllen.

Die Gemeinde, die die Botschaft hinterfragen kann, um sie sich für ihre Situation zu eigen zu machen, wird im Jahr 2000 wiederum von Gott aufgefordert werden, den Brief mit der Offenbarung Seines Willens zu essen und weiterzusagen. In der Nutzung der Kommunikationsmöglichkeiten des 21. Jahrhunderts kann die Gemeinde, kann die Gruppe wieder prophetische Gemeinde im Auftrag Gottes werden.

\section{S U M M A R Y}

Any reflection on Christian Communications in the year 2000 must include the prophetic element. The Prophet, (cf. Ezechiel $2: 8-3: 1$ ) does not seek his mission himself, and his message is not comfortable. Thus Christian Communications resides somewhere between resistance and commitment to the world. A few trends for the future can be discerned: Communications has to become more and more important for the Church if she wants to exist between the communications flow from politics and business. Where the Church herself owns media, she has to respond to the laws of media management. Can she use these secular media at all, and if so, how can she put them to good use? Both inside and outside the community of the faithful the Church will discover the communicating parish and develop it for dialogue. Only within Christian communities communicating with each other can the destructive powers of our present human society be changed and reorientated. Christian Communications, therefore, is not chiefly a mass process, but something enabling the individual to enter into dialogue with many. In using the communications possibilities of the 21 st Century in this way, the parish can once more become the prophetic community in proclaiming the Good News.

\section{RÉSUMÉ}

Toute réflexion sur la communication chrétienne en l'an 2000 doit aussi contenir l'élément prophétique. Le prophète (cf. Ezéchiel 2,8-3,1) ne cherche pas sa fonction, et son message n'est pas facile. Ainsi vit la communication chrétienne entre la résistance contre et la soumission au monde. Quelques directions se dessinent comme prévisions pour l'avenir. La communication devra devenir plus importante pour l'Eglise si elle veut continuer d'exister d côté du flot grandissant de communication en ce qui concerne la politique el l'économie. Là où l'Eglise est elle-même possesseur de mass media, elle devra se soumettre aux lois du menagement des mass dia. Saura-t-elle seulement se servir de ces mass media avec leur législation séculairement empreinte, et si oui, comment? L'Eglise devra, à l'intérieur et d̀ côté de la communauté de foi, découvrir et développer la communauté communicante laquelle soit capable de dialoguer. Seules les communautés chrétiennes communiquant entre elles pourront changer et donner une orientation nouvelle aux valeurs, vouées à la destruction, de notre société actuelle. C'est pourquoi la communication chrétienne n'est pas avant tout un processus de masses, mais un incident qui amène tout un chacun à s'échanger avec un nombre aussi grand que possible. Dans ce sens, dans l'utilisation des possibilités de communication du $21 \mathrm{r}$ siècle, la communauté peut à nouveau devenir une communauté prophétique aux ordres de Dieu. 


\section{RES U MEN}

Cada reflexión sobre la comunicación cristiana en el año 2000 debe contener el elemento profético. El profeta (cfr. Ezequiel 2,8 - 3,1) no busca serlo y su misión no es cómoda. Así la comunicación cristiana vive en el mundo entre la resistencia y la entrega. Como pronóstico para el futuro puede hablarse de varias tendencias. La comunicación tendrá que ser más importante para la Iglesia si quiere mantenerse frente a la corriente de comunicación social. Podrá servirse siquiera de esos medios con sus leyes seculares $y$, en caso afirmativo, cómo? La Iglesia deberá descubrir y desarrollar en la comunidad de creyentes la comunidad comulgante, capaz del diálogo. Solo comunidades comulgantes entre sí podrán alterar y cambiar de rumbo los valores de nuestra sociedad actual, abocados a la destrucción. Por ello la comunicación cristiana no es predominantemente un proceso masivo, sino un fenómeno que capacita al individuo para intercambiarse con muchos. En la utilización de las posibilidades de la comunicación social en el siglo XXI llegará la comunidad a ser comunidad profética por mandato divino. 